\title{
Mice with targeted gene disruptions or gene insertions for diabetes research: problems, pitfalls, and potential solutions
}

\author{
E. H. Leiter
}

The Jackson Laboratory, Bar Harbor, Maine, USA

\begin{abstract}
The mouse has been a favoured organism for molecular manipulation in studies seeking to establish the genetic bases and pathophysiologic mechanisms underlying both Type I (insulin-dependent) and Type II (non-insulin-dependent) diabetes mellitus. Gene targeting and transgenesis are the two powerful molecular technologies used in these endeavours. Interpretation of results generated from such studies, ei-
\end{abstract}

ther entailing an altered phenotype or the absence of a phenotypic change, is not always simple. This review focuses on certain complications inherent in the methodologies, and outlines steps that can be taken to distinguish effects of the genetic manipulation from unexpected contributions from the genetic background. [Diabetologia (2002) 45: 296-308]

Keywords Mice, genetics, mutations, transgenics, gene targeting.
The American poet Walt Whitman (1819-1892) wrote: "A mouse is miracle enough to stagger sextillions of infidels". Favoured as a model organism by geneticists because of a well-characterized genome sharing extensive homology with the human genome, the mouse has recently become more "miraculous" because of revolutionary developments in molecular genetics over the past two decades [1]. Stocks of mice with experimentally-manipulated genomes now represent important tools for research into the aetiopathogenesis of both Type I (insulin-dependent) diabetes and Type II (non-insulin-dependent) diabetes mellitus. Such stocks further provide essential insight into the genetic control of diabetes-related subphenotypes associated with the development of hyper-

Received: 18 September 2001 and in revised form: 29 October 2001

Corresponding author: E. H. Leiter, The Jackson Laboratory, 600 Main Street, Bar Harbor, Maine 04609-1500, USA, email: ehl@jax.org

Abbreviations: cADPR, Cyclic ADP-ribose; hGAD65, human glutamic acid decarboxylase 65; IR, insulin receptor; QTL, quantitative trait loci; RIP, rat insulin promoter; $\mathrm{Tg}$, transgenic; Tm targeted mutation glycaemia. The two technologies driving genome manipulation are transgenesis and gene targeting [2]. It is now remarkably straightforward to alter the expression of specific mouse genes as well as to use mice as surrogate hosts for expression of genes from other genera. These manipulations allow analysis of gene function by ablation of specific genes or their replacement with variant alleles. In addition to silencing expression of a given gene (gene "knock-outs"), a specific gene's product can be over-expressed, under-expressed (by use of anti-sense or ribozyme technology), or the gene's expression can be conditionally activated or repressed by the incorporation of environmentally-cued regulatory elements. With a complete sequence of the mouse genome close at hand, these technologies have become even more important for identifying functions of genes currently known only as "expressed sequence tags" (ESTs). This review will not catalogue the many examples whereby researchers have exploited the new genetic technologies to develop new mouse models of diabetes or to investigate the role of specific genes in the aetiology of well-established diabetes models. Rather, the purpose is to discuss the problems of interpretation associated with the major genomic manipula- 
tions currently used to investigate the role of specific genes in the development of diabetes in mice. Such a discussion is timely because certain caveats associated with the technologies, at least given their current limitations, are often not fully appreciated by researchers when planning experiments or assessing phenotypes.

\section{Transgenic and gene-disrupted mice: basic methods}

Transgenic mice are usually produced by zygotic (micro) injection of cloned DNA into the male pronucleus. The DNA injected can be a specific gene either with an endogenous genomic promoter-enhancer region or a cDNA coupled to a promoter-enhancer designed to produce widespread expression in most tissues. Alternatively, tissue-specific or cell-specific promoters are now commonly ligated to a DNA sequence of interest in order to limit expression to a cell type of choice. Fertilized eggs harvested from hybrid (F1) mice often prove to be more "forgiving" of intranuclear microinjection damage, thereby yielding higher percentages of live-born offspring after transplantation into pseudopregnant "foster" female mice. This, in turn, increases the potential for a higher percentage of transgenic founders. For these reasons, many of the transgenic stocks previously created to answer specific questions in diabetes, aetiopathogenesis have entailed insertion of the transgene into F1 zygotes that yield progeny segregating variable and undefined proportions of genes from both (grand) parental strains.

Transgene insertion can also be accomplished by transduction of totipotent embryonic stem cells in culture using a transgene ligated to a selectable antibiotic resistance marker and then incorporating the transduced embryonic stem cells into a developing embryo at the blastocyst stage. Typically, the blastocyst is obtained from C57BL/6 J (B6) mice whereas the most intensively-utilized embryonic stem cell lines derive from the 129 strains originally developed at The Jackson Laboratory to increase frequency of spontaneous testicular teratomas [3]. Whereas the intranuclear microinjection method will result in random incorporation of tandem copies of the transgene in every cell of the "founder" mice, tissues of mice developing from blastocysts injected with transduced embryonic stem cells will represent chimeric and variable mixtures of cells deriving from the blastocyst donor and from the embryonic stem cell donor genome. For development of a permanent line once a chimeric founder has been identified (usually by chimeric coat and/or eye colour pattern), a "germ-line" transmitting male must be identified and mated. This requires that one or more of the embryonic stem cells injected into the blastocyst follow a differentiation pathway that leads to gamete production in the gonads. In the case of strain 129-derived embryonic stem cells, which derive from male embryos, the stem cell must give rise to sperm. Only if the founder is mated to the identical 129 or other substrain used to generate the embryonic stem cell line will the transgene be coisogenic (genome identical except for the experimentally-mutated allele) on a standardized inbred strain background. Over time, the various strain 129 and " $129 /$ Sv" mice (the latter actually a congenic stock) distributed around the world for the creation of embryonic stem cell lines have diverged genetically $[4,5]$. The basis for this heterogeneity and the nomenclature implemented to differentiate the various substrains with known genetic differences (e.g. 129X1/SvJ, 129S1/SvImJ, 129T2/SvEmsJ, etc.) can be found at http://jaxmice.jax.org/html/nomen clature/129nomenclature.pdf. Whether significant differences in glucose homeostasis distinguish these various substrains has never been examined; however, since minor histocompatibility differences and behavioural differences are distinguishing features, investigators using strain 129-derived embryonic stem cell technology should verify the correct 129 strain of origin.

"Targeted mutagenesis" uses an alternative form of embryonic stem cell technology described above with the major distinction being that the introduced gene is not incorporated at random into the genome. On the contrary, the precise integration site is known since homologous recombination is used to incorporate an altered genomic segment into a specific locus to either "knock-out" a single copy gene or to "knock-in" additional copies of the gene, or an allelic variant, or the endogenous allele containing an inserted marker to allow cell tracking [6]. Although B6-derived embryonic stem cell lines exist $[7,8]$ and embryonic stem cell lines from the autoimmuneprone NOD mice are under development [9], the bulk of the gene-targeted models reported entail the use of strain 129-derived embryonic stem cells. The technique also requires identification of chimeric founder male mice that are capable of germ-line transmission of the targeted gene. As noted above, coisogenicity for the targeted gene on a geneticallystandardized background can only be achieved if the founder is mated to the identical substrain used to generate the embryonic stem cell line.

\section{Type I and Type II diabetes in mouse models}

Type I and Type II diabetes in rodents and in humans are complex diseases in which multiple genes interact with each other and the extrinsic environment to influence disease susceptibility and progression. Welldefined mouse models for both disease types and their complications are powerful tools for systematic identification of the interacting cellular and molecu- 
lar pathways [10]. In both types of diabetes models, there are clear phenotypic "checkpoints" through which a mouse must transit before crossing a pathogenic threshold into overt clinical disease. Prodromal subphenotypes characterizing the progression of diabetes in mice are the same ones conferring a major risk of diabetes in humans. The prodromal phenotypes of Type II diabetes in mice include early weight gain or adiposity often associated with increased metabolic efficiency, hyperinsulinaemia and/or insulin resistance, and impaired glucose tolerance (this triad generally developing in male mice at or after puberty). In mouse models of Type I diabetes, such as the NOD mouse, prodromal subphenotypes include defects in maturation of antigen presenting cells, increases in both cell mediated and humoral immunity to pancreatic beta-cell antigens, development of insulitis, and loss of pancreatic insulin content. Different subsets of genetic polymorphisms predisposing to the development of some or all of these subphenotypes have been randomly fixed (by inbreeding) into the variety of inbred mouse strains now available for biomedical research.

A landmark event in the field of mouse genetics was the discovery that inbred strain background exerted a powerful effect on the diabetogenic potential of single gene obesity-producing mutations [11]. Mutation in either the leptin gene (originally the "obese" mouse, $o b$, now $\left.L e p^{o b}\right)$ or the leptin receptor gene (originally the "diabetes" mouse, db, now Lepr ${ }^{d b}$ ) produced a phenotype of massive obesity with only transient diabetes when studied on the C57BL/6 J (B6) inbred strain background. In contrast, the same two mutations produced massive obesity with a lifeshortening diabetes on the related C57BLKS/J (BKS) inbred strain background [11, 12]. When the genetic basis for such differences in background is analysed, it is commonly found that in new hybrid combinations, both parental strains contribute decompensating quantitative trait loci (QTL) capable of converting obesity to obesity-induced diabetes ("diabesity") $[13,14]$. Similarly, in the NOD mouse model of T cell-mediated Type I diabetes, outcrosses with other strains usually completely suppress diabetes in the F1 generation unless the outcross partner is MHC-identical to NOD and genetically-related [15]. When diabetes does develop after backcrosses to the NOD-inbred strain background, new combinations of non-MHC loci, some derived from the supposedly "resistant" outcross partner strain, apparently contribute to aetiopathogenesis $[15,16]$. Indeed, an interspecific outcross between NOD and Mus spretus provided a striking example of the unmasking of latent diabetes susceptibility genes [17]. As expected, no diabetes was observed in $\mathrm{F} 1$ hybrids given that disruption of the homozygous combination of the NOD strain's MHC and non-MHC alleles (so-called "Idd" loci) suppressed spontaneous autoimmune diabeto- genesis. At first backcross to NOD, wherein some homozygous assortment of diabetogenic NOD alleles could be reassembled, a low frequency of spontaneous hyperglycaemia was observed. Two remarkable features distinguished this hyperglycaemia from the syndrome in standard NOD mice; the frequency of diabetes was higher in male mice $(12 \%)$ than in female mice (3\%) and there was no insulitis associated with this diabetes syndrome. Detailed analysis showed the syndrome to be one of insulin resistance with pancreatic islet hypertrophy and hyperinsulinaemia [17]. An example below emphasizes how undefined genetic admixtures can complicate the interpretation of experiments utilizing putatively "normal" inbred strain backgrounds with genetically targeted alleles or transgenes.

\section{Strain 129: What do we know about glucose homeostasis in these mice?}

Although it is well-recognized by mouse geneticists that different inbred strains harbour different susceptibilities for either Type I or Type II diabetes, a systematic metabolic characterization is lacking for 129 strains, the source of most of the embryonic stem cell lines used in gene targeting [3]. Targeted mutations generated in 129-derived embryonic stem cells are usually injected into B6 blastocysts. The phenotype produced by the "knockout" is often reported for lineages derived from an undefined mix of both B6 and 129 parental genomes. Such chimeric stocks are formally designated as B6;129. In this segregating background, though the targeted mutation could be fixed to homozygosity, the phenotype associated with this mutation could remain highly variable, often seen in only $50 \%$ or fewer of the mice that carry the targeted mutation. This common phenomenon is a clear indication that other genes in the parental strain background are modifying the phenotype in undefined ways. Strain $129 /$ J certainly contains genes that are highly permissive to the development of massive obesity (Fig. 1) and extreme hyperinsulinaemia in homozygotes expressing the Lepr ${ }^{d b-3 J}$ mutation [18]. A previous study analysed the development of overt diabetes and various subphenotypes in progeny produced from an outcross of C57BLKS/J-Lepr db heterozygous females to strain $129 / \mathrm{J}$ males, followed by backcross to C57BLKS/J-Lepr ${ }^{\mathrm{db}}$ heterozygotes to generate homozygous mutants segregating for background modifiers [19]. Polygenic inheritance was indicated, with the assumption made that all deleterious background modifiers must have been contributed by the diabetes-susceptible C57BLKS/J background [19]. However, the use of polymorphic microsatellite markers to trace the contributions of both parental genomes in hybrid crosses frequently detects deleterious contributions from both the susceptible 


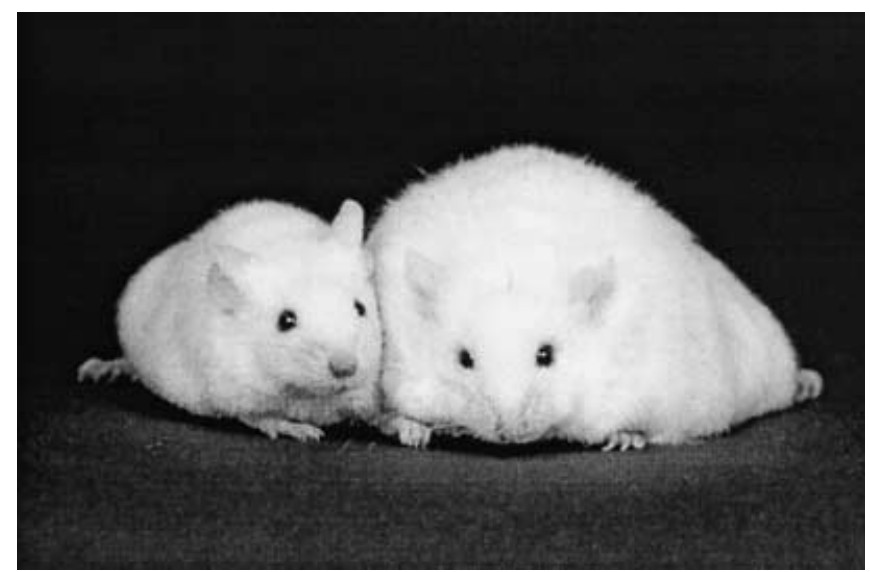

Fig. 1. A homozygous $129 / \mathrm{J}-$ Lepr $^{d b-3 J}$ mutant mouse (right) and littermate control (left) showing the massive obesity phenotype produced on this inbred strain background. The hyperinsulinaemia elicited by this mutation was more extreme than observed on other inbred strain backgrounds expressing other mutant alleles at the Lepr locus

and the putatively resistant backgrounds when new genomic combinations are created.

Genetic disruption of the insulin receptor (IR) gene (Insr, chromosome 8) illustrates the problem of dissociating primary effects of ablating a specific gene function from secondary effects produced by undefined background contributions. Heterozygous mice must be studied because the "knockout" produces a neonatal lethal form of diabetes [20]. Variable hyperinsulinaemia was observed in B6;129 mice heterozygous for the mutation, indicative of phenotypic interference by undefined background genes. After backcross to the B6 parental background, congenic mice heterozygous for the Insr mutation produced only mild hyperinsulinaemia; in contrast, mice heterozygous for the Insr mutation after backcross to the $129 / \mathrm{Sv}$ parental genome showed severe hyperinsulinaemia and/or insulin resistance [21]. The greater severity of the hyperinsulinaemia and/or insulin resistance syndrome observed on the 129 inbred background, consistent with that produced by expression of the Lepr ${ }^{d b-3 J}$ mutation on the 129/J background, reinforces the notion that the 129 strain genome harbours latent, undefined diabetes susceptibility quantitative trait loci (QTL) capable of enhancing the deleterious effects of reduced IR signalling. Five QTL contributing to the phenotype were identified in progeny of an intercross between the B6 congenic stock heterozygous for the disrupted Insr gene and the $129 / \mathrm{Sv}$ congenic stock heterozygous for the same mutation. Of these, four deleterious QTL were contributed by the seemingly hyperinsulinaemia-resistant B6 background, with only one deleterious QTL identified from the hyperinsulinaemia-permissive $129 / \mathrm{Sv}$ background [21]. This analysis was limited to males because females did not develop hyperinsulin- aemia. Such male sex-specific effects are frequently encountered in Type II diabetes mouse models [22] and complicate transgenic or gene targeting experiments if the data are not stratified by sex. These results emphasize the problem of relating the effect of a particular targeted gene mutation on a segregating B6;129 mixed background wherein both parental backgrounds can contribute to metabolic dysfunction in undefined ways. They certainly provide insight as to why only about $40 \%$ (rather than $100 \%$ ) of B6;129 mice doubly heterozygous for a disrupted Insr gene and a disrupted insulin receptor substrate1 encoding gene (Irs 1) developed an insulin resistance syndrome [23]. This variability in the development of a diabetic phenotype is well-illustrated in a more recent report where triple heterozygotes with targeted genes encoding IR, IRS-1 (Irs1, chromosome 1), and IRS-2 (Irs2, chromosome 8, positioned $4 \mathrm{cM}$ distal to the Insr locus) were assembled on a mixed background [24]. Thus, the genetic environment must be standardized if the specific metabolic contribution of a mutation to diabetogenesis is to be separated from undefined strain background contributions.

The phenomenon of unanticipated insulin resistance being generated when two unrelated genomes are combined (with both parental strains apparently showing normal insulin sensitivity) is well-known to mouse geneticists. Examples of this include the spontaneous development of hyperinsulinaemia and IGT in $(\mathrm{C} 3 \mathrm{H} \times \mathrm{I} / \mathrm{Ln}) \mathrm{F} 1$ male mice [25], (B6 x BTBR $) \mathrm{F} 1$ male mice [26], C3H.SW male mice [27], and (NZO $x$ NON)F1 male mice [13]. As indicated in the example of the targeted IR gene mutation discussed above, the 129 strain genome certainly has underlying metabolic peculiarities that could "uncover" to enhance insulin resistance in a rather dramatic manner. A strongly diabetogenic contribution from the 129T2 genome was uncovered in an outcross carried out at The Jackson Laboratory to identify QTL associated with seizure susceptibility. The partner strain used in this outcross was EL/Suz, known for genetic susceptibility to experimentally-induced epileptic seizures [27, 28]. Strain 129T2 is seizure-resistant. Following EL x 129T2 outcross, a first backcross (BC1) to EL was carried out. The surprising observation was that $\mathrm{BC} 1$ of both sexes were becoming markedly obese. The author received these mice for analysis of serum immunoreactive insulins (IRI) and pancreatic histopathology. In strain 129T2 parental mice of both sexes and in EL/Suz female mice, serum IRI concentrations were normal $(<2 \mathrm{ng} / \mathrm{ml})$. A pool of EL male serum showed moderately increased IRI $(5.6 \mathrm{ng} / \mathrm{ml})$. The obese $\mathrm{BC} 1$ mice of both sexes exhibited extreme hyperinsulinaemia (serum IRI $>50 \mathrm{ng} / \mathrm{ml}$ ); furthermore, $\mathrm{BC} 1$ male mice, but not female mice were overtly hyperglycaemic (plasma glucose, PG, $>22 \mathrm{mmol} / \mathrm{l}$ by 12 weeks). The pancreatic histopa- 

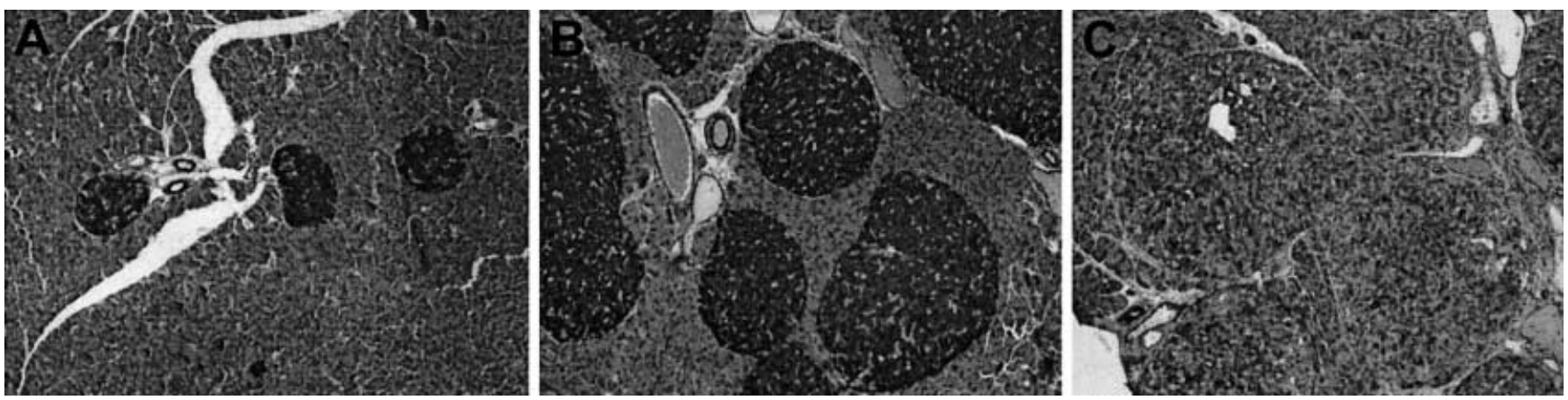

Fig. 2. Unmasking of latent diabetogenic susceptibility when "normal" $129 / \mathrm{Sv}$ mice are outcrossed with EL/Suz mice, followed by one backcross of the phenotypically-normal F1 hybrid backrossed to the EL/Suz strain. Pancreatic histopathology is shown using an aldehyde fuchsin stain for granulated beta cells. A normal EL male islets B well-granulated hyperplastic BC1 female islets $\mathbf{C}$ hyperplastic but markedly degranulated islets from a diabetic BC1 male

thology of BC1 male and female mice is shown in Figure 2. Massive islet hypertrophy and hyperplasia equivalent to that originally seen in strain $129 \mathrm{P} 3 / \mathrm{J}$ mice homozygous for the coisogenic $\operatorname{Lepr}^{d b-3 J}$ mutation [18] was observed in both sexes. Compared to islets from $\mathrm{BC} 1$ female mice, the beta cells of the diabetic $\mathrm{BC} 1$ male mice were highly-degranulated, correlating with their failure to compensate for the hyperglycaemic stress.

Unexpected contributions from the 129 genome interacting deleteriously with B6 genome very likely revise the interpretation of hyperglycaemia reported in older "mice" homozygous for a disrupted 32 -microglobulin $(\beta 2 \mathrm{~m})$ gene in a segregating B6;129 chimeric stock [29]. This maturity-onset loss of glucose homeostasis was proposed to be autoimmune in aetiology. However, this conclusion was refuted by many other laboratories that transferred the disrupted $\beta 2 m$ allele onto the autoimmune-susceptible NOD inbred strain background [30-32]. The absence of MHC class I molecules on cell surfaces resulting from the absence of B2-microglobulin, and the attendant failure to develop a CD8 T cell repertoire, abolished rather than triggered autoimmune diabetes. This implicated B6;129 genetic background admixture rather than the presence or absence of B2-microglobulin and CD8 + T cells as the basis for the previously reported "autoimmune diabetes". Indeed, when a group of 9 female and 30 male B6;129-ß2-microglobulin-deficient homozygotes (between ages 3-22 months) were examined for hyperglycaemia in a specific pathogen-free facility at The Jackson Laboratory, none were hyperglycaemic but 3 older male mice had developed marked hyperinsulinaemia ( > $10 \mathrm{ng} / \mathrm{ml}$ ) consistent with insulin resistance, and not autoimmune diabetes [30]. Extensive pancreatic islet hypertrophy with variable beta-cell degranula- tion, a characteristic of insulin-resistant mice, was observed in both ageing B2-microglobulin-deficient B6;129 mice as well as wild-type/“knockout" heterozygotes provided by Dr. L. Wicker from her colony at Merck Laboratories, Rahway, NJ. Pancreata from both genotypes showed variable peri-insulitis, but neither genotype exhibited intra-islet lymphocytic infiltration. Failing to control for potential diabetogenic interactions inherent to different combinations of B6 and 129 genes very likely could account for numerous discrepancies among different laboratories studying the same targeted mutation (for example, "knockouts" leading to total ablation of gene expression [33]). Extended culture of embryonic stem cell lines leads to the accumulation of chromosomal aberrations that not only reduce the germ line competency of the cells, but also could generate phenodeviants independent of the targeted mutation induced in a given line should they be transmitted. These examples show the need to exclude the 129 genome through controlled backcrosses, leaving only the minimum strain 129-derived segment flanking the targeted allele if effects of a specific mutation on development of insulin resistance and IGT are examined.

\section{Know your genetic background and exclude linked 129 genome}

Traditional congenic strain preparation alone could be insufficient to ascertain that the observed phenotype results from the introduced genetic alteration. Even when the genetic background is standardized and the wild-type phenotypic parameters are well-established, investigators must bear in mind that other linked genes in the segment of strain 129 , other than the targeted allele of interest, could be responsible for any altered phenotype that is observed. When a targeted mutation is introgressed into a defined inbred strain background over multiple backcross cycles, an investigator might commonly type only for the (heterozygous) presence of the disrupted allele, frequently by PCR detection of an antibiotic resistance cassette (e.g., neo, neomycin resistance) introduced into the sequence. Statistically, more than $99 \%$ of the genome should be of recipient origin after 
B6.129P2-Cd38 $8^{\text {tm1Lnd }}$

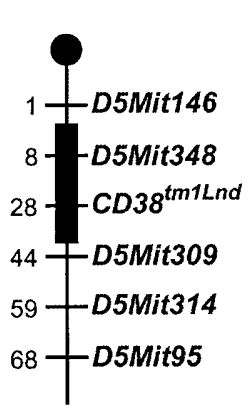

Glucose tolerance test

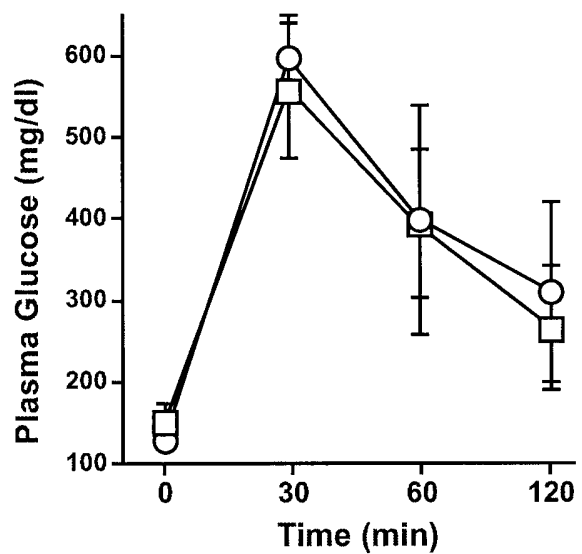

Fig. 3. Length of the $129 / \mathrm{Sv}$ congenic segment (in bold) on Chromosome. 5 after 10 backcrosses to B6, with selection only for a targeted (neo-containing) $C d 38$ gene. The line graph compares glucose tolerance of $10^{\text {th }}$ backcross CD38-deficient male mice $(\bigcirc)$ and standard B6 males $(\square)$ and shows no significant differences ( $n=3$ per group, 16 weeks of age)

ten backcrosses. However, statistics will not accurately predict the extent to which genes linked to the targeted allele are removed by recombination as introgression proceeds. When single marker selection is the sole basis for congenic construction, very long congenic segments are often retained. For example, we analysed the length of the congenic segment on chromosome 5 bearing a disrupted $C d 38$ allele (from 129 embryonic stem cells) received after the targeted $C d 38$ had been backcrossed 10 times onto the B6 inbred strain background [34]. The minimum length of the congenic segment is more than $20 \mathrm{cM}$, approximating a quarter of this chromosome (Fig. 3). The gene product, $\mathrm{CD} 38$, is an ubiquitously-expressed NADase with the ability to generate cyclic ADP-ribose (cADPR); cADPR was proposed to be a key intermediate in calcium-dependent signalling pathways that control glucose-stimulated insulin secretion in pancreatic beta cells [35]. A previous study on the effect of a targeted $C d 38$ gene which was backcrossed only several times to CD-1, an outbred strain, reported impaired glucose tolerance in CD38-deficient homozygotes [36]. This led to the conclusion that CD38 was required for normal beta-cell secretory function. However, glucose tolerance was completely unaffected by the absence of CD38 (as verified by flow cytometry of leukocytes) in the $10^{\text {th }}$ backcross B6 stock at The Jackson Laboratory (Fig. 3). It is plausible that variability in the number of genes retained in linkage to the targeted allele from the embryonic stem cell donor strain could have contributed to the inter-laboratory variation. The solution to the linked neighbour problem is to use polymorphic genetic markers to select breeders at each backcross cycle that are homozygous for the recipient strain's marker alleles both above and below the donor 129 strain allele being introgressed. Several web sites provide such information, including that of the Center for Inherited Disease Research (http://www.cidr.jhmi. edu/main.html) and also available by request from the Allele Typing Facility at The Jackson Laboratory (http://www.jax.org/jax/sss/micro_chem/alleletyping/ strainsurvey).

Assuming in the example above that segments of chromosome 5 of differential length were carried into the two separate congenic stocks, it is equally likely that the markedly different genetic backgrounds on which the targeted gene was compared also contributed appreciably to the different phenotypes. The outbred CD-1 (or ICR "Swiss") genome is the origin of inbred strains with known impairment of glucose tolerance and propensity to Type II diabetes, including NON, NSY, and ALS [13, 37, 38]. Hence, unidentified genes from CD-1 mice predisposing to impaired glucose tolerance and beta-cell function could have interacted with the deficiency of CD38 to impair glucose tolerance. Indeed, glucose tolerance tests in CD-1 background mice segregating for the wild-type $C d 38$ allele showed poor glucose clearance kinetics [36]. This was true even after the mice homozygous for the targeted $C d 38$ allele were crossed with wild-type mice carrying a human $C D 38$ gene on a rat insulin promoter. To backcross targeted mutations to $\mathrm{CD}-1$ outbred mice is attractive because of their superior reproductive performance, low cost, and ability to sustain deleterious mutations that reduce viability or fecundity in highly-inbred strains. However, this practice carries risks for analysis of phenotypic changes associated with diabetes-related parameters such as glucose tolerance or insulin sensitivity. The phenotypic variability in such outbred mice was shown in a study [39] where body weights of 10 to 12 -month old CD-1 female mice was found to vary between $35 \mathrm{~g}$ and $55 \mathrm{~g}$. Serum leptin concentrations showed a tenfold difference between the high and low weight extremes.

Linked genes with potential immunomodulatory effects have proven particularly problematic in studies wherein specific gene deficiencies engineered in strain 129 embryonic stem cells have been introgressed into the NOD genome to assess effects on the development of Type I diabetes. For such applications, it is essential not only to know the chromosomal location of the targeted allele but also what other strain 129 genes in linkage have been carried over. For example, assume an investigator is interested in the effect on NOD diabetogenesis of targeting the gene encoding Stat1 (Signal transducer and activator of transcription 1), a protein important in mediating signalling from the interferon gamma (IFN $\gamma$ ) receptor. The Stat1 gene is on chromosome $1(\sim 25.9 \mathrm{cM})$, sited just outside a region containing multiple "Idd" loci [40]. Hence, unless the congenic segment in a gene targeting experiment were limited to only a very 
short segment of strain 129 genome flanking Stat1, it would be exceedingly difficult to assign phenotypic changes in the diabetogenic process simply to the loss of Stat1 gene function in mediating IFN $\gamma$ signalling. In this hypothetical targeting experiment, it would not be advisable to use strain 129-derived embryonic stem cells or any embryonic stem cells not derived from the NOD strain itself. Unfortunately, NOD embryonic stem cells are not yet available but efforts are underway to develop such lines [9]. Until germ-line competent NOD embryonic stem cells become available, the investigator must be aware of where in the genome the gene of interest resides. As discussed above, informative polymorphic genetic markers distinguishing NOD from the strain donor of the embryonic stem cells (generally from 129 substrains) should be used at each backcross cycle (to NOD) to eliminate as many as possible of both the linked and unlinked strain 129 alleles segregating at each backcross generation.

The problems created by "linked neighbours" is well illustrated by attempts to assess the role of IFN $\gamma$ itself in NOD diabetogenesis. When a targeted mutation in the structural gene for IFN $\gamma$ was introgressed into the NOD genetic background, diabetes still developed [41, 42], suggesting that this prototypic T-helper 1 cytokine was not absolutely essential for the development of Type I diabetes in the model. However, contradictory results were subsequently reported, showing that a targeted mutation in the alpha chain of the IFN $\gamma$ receptor prevented Type I diabetes when introgressed into NOD [43]. Subsequent subcongenic analysis of the segment of chromosome 10 containing the disrupted receptor gene resolved the conundrum. It was not the disrupted receptor that diminished Type I diabetes but a heretofore unidentified $129 / \mathrm{Sv}$-derived resistance locus in linkage to the mutation [44]. This conclusion was buttressed by the report that transfer into the NOD strain background of a targeted mutation in the IFN $\gamma$ receptor beta chain failed to prevent Type I diabetes [45].

If one observes a phenotypic change apparently due to the disruption of a specific allele such as a cytokine-encoding gene, and the wild-type gene product is available as a recombinant protein, the direct approach to show that the phenotypic change was elicited by the targeted allele and not by other linked genes, is to treat the "knock-out" animals with the recombinant protein. Such manipulations should reverse the altered phenotype if the deviant phenotype is completely attributable to the mutation. This was done, for example, to show that the juvenile-onset colitis in $\mathrm{C} 3 \mathrm{H} / \mathrm{HeJBir}$ mice congenic for a disrupted Il10 allele (chromosome 1) was indeed triggered by IL-10 deficiency and not by other endogenous modifiers [46]. More difficult is the reintroduction of a wild-type allele through gene therapy or via "knockin" of a wild-type allele into the embryonic stem cell line carrying the targeted mutation followed by the development of a new stock of mice from these cells.

\section{Gene redundancy and phenotype assessment in gene targeted mice}

Two issues were raised in the preceding section. These were (i) the critical importance of genetic background definition before making phenotypic comparisons between gene-intact versus gene-disrupted sibs and (ii) the possibility that strain 129 genes linked to the disrupted allele could contribute to, and in some cases, be responsible for phenotypic differences. It can thus be difficult to interpret gene targeting experiments in which disruption of a specific allele fails to demonstrably alter a diabetogenic process (e.g. the absence of IFN $\gamma$ failing to prevent Type I diabetes development in NOD), or reciprocal$1 y$, if the loss of function fails to induce a predicted impairment in glucose homeostasis (e.g. muscle cellspecific deletion of the IR) [47]. This latter observation was quite unexpected given the central role assigned to muscle insulin resistance in the pathogenesis of human Type II diabetes. This result might reflect differences between the two genera or, as discussed elsewhere, could represent the ability of the genome to metabolically compensate for some deficiencies [48]. A more recent and extensive analysis of insulin sensitivity of these "knockout" mice indicated that a primary defect in muscle glucose transport produces secondary defects in insulin action in adipose tissue and liver due to glucose toxicity [33].

There are numerous instances to show the ability of the genome to compensate for loss of certain genes by upregulating related genes in genetically engineered mouse mutants. Before gene targeting of the polyADP ribose polymerase-1 encoding gene (Adprt1, Chromosome. 1) "knockout", it was assumed that a single gene encoded this unscheduled DNA repair enzyme. Removal of this major activity by gene targeting revealed unrecognized familial redundancy by showing that "global" PARP activity measured in a damaged cell represents contributions from at least five distinct but related enzymes [49]. In mice with targeted mutations in the neuronal nitric oxide synthase gene (Nos1 chromosome 5, $5 \mathrm{cM}$ ), there is a $20 \%$ increase of the activity of the endothelial form (Nos3, chromosome 5,65 cM) [50]. Very likely, the failure to identify an effect on glucose homeostasis or growth associated with the IRS-3 "knockout" mouse [51] could relate to compensation produced by the other IRS-encoding genes. These examples underscore the fact that gene targeting experiments analyse the effects of the missing gene product rather than the effects of the gene product directly. To extrapolate the import of this to the controversy regarding the role of IFN $\gamma$ in NOD diabetogenesis, the finding that Type I diabetes develops in 
NOD stocks lacking either IFN $\gamma$ or the ability to respond to it does not mean that IFN $\gamma$ itself is unimportant in the natural history of spontaneous diabetes development in this mouse model.

\section{Control of the physical environment}

Tight control of the physical environment must also be maintained by investigators applying transgenesis and "knockout" technologies. In genetic modifications expected to impair carbohydrate and/or lipid homeostatic mechanisms, the dietary macronutrient content often requires manipulation in order to discern the consequence of the genetic change. When toxin-bearing transgenes driven by the Uncoupling Protein-1 (Ucp1) promoter are used to ablate brown adipose tissue, the relatively low fat content of the rodent chow diet $(6.5 \%)$ must be increased to "Western" amounts $(21 \%)$ to assess the diabetogenic contributions of this adipose tissue-specific ablation [52]. Additional variables in the physical environment that must be controlled are caging densities, segregation of the sexes, and room temperature and humidity. In studies on Type I diabetes using the NOD mouse, a specific pathogen-free and rigidly-controlled environment must be maintained to separate effects of the experimental manipulation from uncontrolled variables [53,54]. This is because the penetrance of Type I diabetes in NOD mice of both sexes can be drastically and variably suppressed by the exposure of these mice to a broad spectrum of microbial agents and endocrine factors [55]. The protective effect reported to result from a given genetic manipulation in a colony of NOD mice wherein the "wild-type" incidence is only $50 \%$ in female mice by 30 weeks of age might not be observable if the manipulated stock is rederived into a vivarium wherein the frequency of Type I diabetes in female mice by 20 weeks of age exceeds $80 \%$.

\section{Neighborhood effects}

A commonly used procedure of disrupting a gene involves the insertion of a drug selection cassette into an exon. Instances have been reported where such molecular manipulations have not only produced a null allele in the gene intended, but also produced significant cis-effects on adjacent genes. This effect usually is reported in related genes in tandem that have evolved joint locus control elements in cis configuration, such as a cluster of genes encoding myogenic transcription factors on chromosome 7 [56]. The importance of vector design is shown in a study where a DNase hypersensitive site in the locus control region of the $\beta$-globin gene was targeted with a $P G K / n e o$ cassette. The targeted mutation produced appreciable changes in $B$-globin gene expression, but subsequent removal of the $P G K /$ neo cassette from the construct, restoring normal transcription, showed that the effect was due to the inserted selection cassette and not to the deletion [57]. For these reasons, many investigators now design their vectors such that a stop codon is engineered into the targeting sequence and the selection cassettes are flanked by sequences that allow for their removal in embryonic stem cells or in vivo by means of yeast Flp or bacteriophage Cre recombinases [58]. As will be discussed below, the Cre/lox $\mathrm{P}$ procedure of making cell specific "knockouts" could also be subject to artifacts independent of the targeted allele's function.

It is surprisingly common to observe no gross phenotypic effect after the disruption of a gene of undoubted relevance to a life process. The preceding discussion emphasized the advisability of assessing phenotypes on several different inbred backgrounds. Additionally, a variety of different tissues should be analysed. For example, a targeted mutation in a regulatory intron in the collagen 1 locus had no effect on constitutive expression in the skin but the mutation decreased expression in the lung in an age-dependent fashion [59]. Indeed, the effects of a number of targeted mutations expected to affect lipid or glucose homeostasis or body mass regulation have been subtle, often requiring a combination of extended longitudinal analysis and sophisticated physiogenomic studies. The effect of a peroxisome proliferator-activated receptor gamma "knockout", expressed in the heterozygous state to increase insulin sensitivity, required hyperinsulinaemic euglycaemic clamp analysis in 8-month-old male mice to identify an unexpected increase in insulin sensitivity [60]. The fact that mice carrying targeted mutations in the homozygous state are born and weaned in normal numbers could be deceiving. The first report of "normal in vivo immune responses" in weanling IL-2 "knockout" mice [61] missed the massive lymphoproliferative and inflammatory bowel disease developing in older mice [62].

\section{Transgenesis}

A well-known American college football coach once opined that three things could happen to a team when a pass was thrown, and two of these were bad (a poorly-thrown pass might be dropped by the receiver or intercepted by the opponents). An aspect of the "errant pass" concept is relevant to the procedure of transgenesis. The transgene construct could integrate into a portion of the genome where it cannot be expressed, or alternatively, it could integrate into another gene, thereby inactivating it ("insertational mutagenesis"). An excellent example of the latter ensued from an effort to reduce beta-cell cAMP concentrations by transgenic expression of a yeast cAMP phosphodiesterase gene (Sra5) driven 
by an insulin promoter [63]. Among the five transgenic lines produced, one line was T-lymphocyte and B-lymphocyte immunodeficient. It was subsequently discovered that the transgene integrated into the protein kinase, DNA dependent, catalytic subunit (Prkdc) locus on chromosome 16. This essentially created a new insertational mutation similar to the well-known and immunodeficiency-producing Prkdc $c^{\text {scid }}$ mutation. To circumvent these "errant passes", multiple lines of transgenic mice should be produced and analysed since transgene insertion is essentially random and each line usually contains different transgene copy numbers. Comparison of multiple lines is essential for determining whether a transgene's effect is an intrinsic property of its function, or instead represents insertational mutagenesis or high copy number-generated phenomena.

Differences in transgene copy number are often important determinants of phenotype, especially when MHC genes are introduced. Development of Type I diabetes through beta-cell failure without autoimmune aetiology is frequently observed when rat insulin promoters are used to drive heightened expression of a variety of gene products, including, but not limited to MHC class I or class II molecules [64] in mouse beta cells. In a study wherein $\mathrm{H} 2$-Ea expression was transgenically restored in antigen presenting cells of nominally $\mathrm{H} 2$-Ea non-expressing NOD/Lt mice, complete diabetes protection was observed in a NOD/Lt "high" copy number line transgenically expressing I-E molecules at a higher concentration than $\mathrm{BALB} / \mathrm{c}$ mice, a constitutively $\mathrm{H} 2$-E expressing strain [65]. A lower copy number line expressing I-E molecules at a concentration comparable to $\mathrm{BALB} / \mathrm{c}$ was only partially protected.

Copy number alone does not equate with expression concentration; the site of integration can also be a critical determinant and should be established whenever possible. For example, in a study wherein NOD/Lt zygotes were injected with a human GAD2 transgene (encoding glutamic acid decarboxylase 65, hGAD65) driven by an insulin promoter, two transgenic lines were produced. In one line (A-line), the transgenes disrupted a locus on chromosome 15 required for normal development. Hemizygous mice were born with 4 to 5 copies of the transgene. In another line (Y-line), about 17 copies of the transgene integrated on the Y chromosome. These male mice were also functionally hemizygous. Despite the higher copy number in the $\mathrm{Y}$ line, the A-line expressed higher concentrations of hGAD65 mRNA, immunoprecipitable hGAD65 protein, and GAD enzyme activity [66]. Only mice in the A-line were protected from diabetes, with further experiments suggesting that the site of transgene integration rather than the amount of transgene expression was the more important determinant since protection did not entail development of tolerance to GAD [66].
Many transgenic facilities are accustomed to inject transgene constructs into zygotes from hybrids rather than directly into NOD zygotes, although the investigator may want to assess the effect of the transgene on the diabetogenic process in NOD mice. Use of older NOD female mice as embryo donors, and reducing the concentrations of gonadotropins injected permits successful transgenesis directly into NOD zygotes [67]. Direct injection of transgenes into NOD zygotes avoids ambiguities when the transgene must be introgressed from a hybrid genetic background. Such ambiguity is shown in a report in which GAD antisense transgenes ligated to an insulin promoter were introduced into (B6 x SJL)F2 mice and then backcrossed into NOD mice [68]. Three lines were produced, with varying expression of the antisense construct. The highest expressing line was completely protected from insulitis and diabetes. An unusual feature of this resistant line was the finding that it also was resistant to adoptive transfer of diabetes by splenic leukocytes from overtly-diabetic nontransgenic NOD mice. Since splenic T cells from overtly diabetic NOD mice are known to respond to a multiplicity of beta-cell autoantigens, a possible explanation for this remarkable resistance is that the transgenic line was not histocompatible with standard NOD mice (due to the presence of chromosomal segments derived either from B6 or SJL) such that diabetogenic $\mathrm{T}$ cells could not engraft. One might also speculate that reduced production of the GAD reaction product, gamma aminobutyric acid (GABA), could have affected T-cell function since T cells have GABA-A receptors [69].

Unanticipated immune responses can certainly be generated when genetically-manipulated NOD mice are compared to standard NOD mice. In addition to insulitis and Type I diabetes, NOD mice can develop a broad spectrum of other tissue-specific inflammatory lesions or neoplasms [54]. Among these pathologies is neuritis, inducible in the central nervous system by pertussigen administration [70] and in the peripheral nerves by congenic introduction of a disrupted $C d 86$ allele (chromosome 16) encoding the B7-2 costimulatory molecule [71]. In the latter study, the $C d 86$ deficient NOD stock was found to be Type I diabetes resistant. Logically, this result was interpreted to mean that the induced mutation itself redirected autoimmunity. However, involvement of a gene or genes in linkage to the strain 129-derived $C d 86$ allele on chromosome 16, potentially contributing an allotypic variant from the allele normally expressed in NOD, could also have contributed. For example, the Gap43 gene encoding GAP43 (neuromodulin), expressed exclusively in nerve tissue and associated with regenerative processes is located $2.6 \mathrm{cM}$ distal to the targeted $C d 86$ allele. It is possible, therefore, that neuritis developing in peripheral nerves could have entailed more than loss of B7-2 signalling. Reduction of the congenic segment to 
replace the strain 129-derived Gap43 allele with the NOD allele would be required to eliminate a potential contribution of this locus.

Even if the investigator uses a 20 backcross generation stock in which the congenic segment from a non-NOD donor genome can be shown to be reduced to less than one $\mathrm{cM}$, there is yet one other unanticipated immune response that the investigator should consider, especially when adoptive tissue or cell transfers are part of a study. If the genetically-manipulated mouse lacks a specific antigen or a whole class of antigens, the mutant stock might not be tolerant to adoptively-transferred cells from wild-type NOD mice expressing the relevant antigen or antigens. This was indeed the case when B-lymphocyte reconstitution was attempted in B-lymphocte-deficient NOD/Lt mice congenic for a targeted mutation in the immunoglobulin heavy chain locus [72]. Stable engraftment with wild-type B-lymphocytes required that B-lymphocyte-deficient recipients be lethally irradiated and chimerized with a mixture of mutant bone marrow and purified wild-type B lymphocytes to establish tolerance. Without this manipulation, $\mathrm{T}$ lymphocytes in the genetically-altered stock rapidly rejected adoptively transferred B-lymphocytes [72]. This finding certainly suggests that investigators pursuing adoptive transfer studies involving stocks carrying genetically-targeted null alleles should be concerned about T-lymphocyte tolerance issues.

\section{Transgenesis entailing the Cre/loxP system of site-specific recombination}

Cre is a bacteriophage $\mathrm{P} 1$ recombinase cutting out DNA between two 34 bp loxP sites introduced into a targeting construct for embryonic stem cell mutagenesis. When transgenic mice are produced in which Cre expression is controlled by ligation to a tissue-specific or conditional promoter, such mice can be crossed with other mice carrying a recombinant gene of interest (derived from embryonic stem cells) that contains exons flanked by loxP sites. Tissue and/or cell-specific expression of Cre elicits excision of the DNA between the flanking loxP sites. Where a "global" knockout produces a lethal development, a tissue-specific Cre-mediated deletion often results in a sub-lethal defect as shown for deletion of glucokinase in the liver [73] or of insulin receptor in muscle and beta cells [74]. However, certain considerations should be kept in mind when the Cre-loxP technology is applied to diabetes models. First, if the promoter used to drive Cre expression is "leaky", an altered phenotype might not reflect a cellspecific event. Care must also be taken that Cre expression in a given cell type is uniform and not mosaic. This can be established by analysing the results of test-crosses between the Cre transgenic stock and other mouse stocks carrying lox $P$-flanked reporter genes such as en- hanced green fluorescent protein (EGFP) or ROSA26. Evidence of mosaicism could be indicated by differences in recombination efficiency when different Cre transgenic lines produced from the same construct are compared [75]. An unanticipated result of transgenic Cre expression could be "illegitimate" Cremediated recombination of endogenous DNA sequences in the absence of introduced loxP sites [76]. High copy number Cre expression can produce endonuclease activity sufficiently high to affect cellular functions [77]. Such a phenomenon could account for our preliminary observation that four lines of transgenic NOD/Lt mice produced at The Jackson Laboratory, all homozygous for the same RIP-Cre construct, but expressing at varying concentrations in beta cells, show reduced frequencies of Type I diabetes in the absence of any other manipulations. Another consideration associated with conditional mutagenesis that is often overlooked is the fidelity of promoter expression driving expression of the Cre recombinase. If the transgene should lack some unknown regulatory sequence present in the endogenous promoter, Cre expression might not exhibit the kinetics or site-specificity desired by the investigator. As noted above, if the lox P-containing gene must be backcrossed into the NOD or other inbred background following homologous recombination in a strain 129-derived embryonic stem cell, the possibility also exists of introducing phenotype-altering genes in linkage to the loxP-flanked gene of interest, even after multiple backcross generations. In studies which modify autoimmune diabetes in NOD mice, this latter problem will be overcome by the eventual development of NOD embryonic stem cell lines for isogenic gene targeting experiments.

\section{Summary}

The ease by which the mouse genome can be experimentally manipulated makes the mouse invaluable for dissecting the pathogenetic mechanism underlying both Type I and Type II diabetes in humans. Detailed discussion of all the uses in murine diabetes research to which recombinant DNA technologies can be or have been effectively applied is beyond the scope of this review. Innovative modifications of these technologies continue to appear, such as the "knock-in" of functional cytokine genes ligated to jellyfish green fluorescence protein so that T cells producing the cytokine can be tracked in vivo and in vitro [6]. Also, the inclusion in a transgene of a bacterial tetracycline transactivator (tTA) or reverse (rtTA) sequence permits temporal regulation of gene expression or excission by the application of doxycycline in the drinking water [78]. This brief review has stressed potential pitfalls and artifacts that can complicate the interpretation of experimental results. In gene targeting experiments, the genomic map posi- 
tion of the gene to be targeted must be considered critically. With the bulk of the mouse genome sequence available in both public and private (Celera, Rockville, Md., USA) databases, acquisition of this information no longer requires the investigator to undertake complicated mapping studies using DNA panels from segregating populations of mice or using mouse-hamster hybrid cell lines. This genome "positional" information is as important to the interpretation of experimental results as is the knowledge of the exon and/or intron structure of the gene to be targeted. Such 'positional' information is equally important in studies utilizing the NOD mouse wherein transgenes are not introduced directly into this genome but are rather introgressed from Type I diabetic-resistant hybrid stocks. Generating a "knockout" mouse or multiple lines of transgenic mice is the laborious first step in a long analytic pathway. Given the commitment of time, effort, and resources required, experiments should be planned to minimize the pitfalls outlined in this review.

\section{A note about nomenclature}

Whenever possible throughout this review, a verbal description of genetically altered mouse stocks has been used in preference to the formal gene designations. The reason is that, because many readers will not be familiar with current nomenclature conventions, clarity is better achieved by textual explanation. However, investigators publishing reports of genetically altered mice should, in their Methods section, use formal nomenclature at first mention of a new stock produced by transgenesis or gene targeting and then denote the "shorthand" descriptor they will use throughout their publication. Nomenclature guidelines and locus registry information for mice carrying transgenes (Tg) or targeted mutations (tm) are available at http://www. informatics.jax.org/mgihome/nomen. Transgenes are now designated simply $\mathrm{Tg}$ (previously, they were prefixed $\mathrm{TgN}, \mathrm{TgH}$ or $\mathrm{TgR}$ to denote their mode of incorporation). The formal designations for the author's two stocks of NOD/Lt mice derived from NOD zygotes directly injected with a construct from which the human gene encoding GAD65 is selectively expressed in beta cells by means of a ligated rat insulin promoter are NOD-Tg (RipGAD2) 1Lt (the line with the transgenes integrated into the Y chromosome); and NOD-Tg (RipGAD2) 2Lt (a second line with the same construct autosomally integrated into chromosome 15). Once the transgenic line has been introduced in full formal regalia, the investigator can then devise shorter or more obvious descriptors (e.g. NOD-hGAD65-Y line and NOD-hGAD65-A line). Likewise, the correct descriptor for a NOD stock congenic (and homozygous) for a targeted mutation in the 32 -microglobulin gene would be NOD.129P2(B6)-B2 $m^{t m 1 U n c}$. The 129P2 descriptor indicates the embryonic stem cell source (129P2/OlaHsd, formerly 129/ OlaHsd. For an explanation of current 129 strain nomenclature, see http://jaxmice.jax.org/html/nomenclature/129nomencla ture.pdf) and the (B6) notation indicates that the mutation was transferred onto its current (NOD) background from a stock derived at least in part from C57BL/6. The gene symbol, in italics, denotes this as the first targeted mutation (superscripted $t m 1$ ) at the $B 2 \mathrm{~m}$ locus generated at the University of North Carolina (superscripted $U n c$ ). After listing the formal descriptor, the author would then introduce his/her shorthand notation (e.g., NOD.B2m null , NOD.B2m $m^{-/}$, etc.). Use of correct nomenclature can indicate, at a glance, important variables that, if recognized, might explain discrepancies between published reports from different laboratories that have targeted or inserted the same gene and have observed different phenotypes. Unfortunately, gene nomenclature itself changes with alarming frequency. Readers of this review will easily infer that Glut4 is the gene symbol for the GLUT-4 transporter. What would they infer about Slc2a4, the more recently assigned symbol denoting solute carrier family 2 (facilitated glucose transporter), member 4 (a.k.a., GLUT-4)? Given the complexities of a changing nomenclature, it is not surprising that many, if not most, of the descriptions of genetically-manipulated mouse stocks reported in the literature do not conform to current genetic nomenclature rules.

Sources. The literature selected for citation by the author represents only a small sampling of published papers describing genetic manipulation of mice for diabetes research. Because the author's objective was to provide perspective rather than review of a field, citations were chosen for their heuristic value.

Acknowledgements. The author is indebted to Staff colleagues, Drs. D. Serreze, L. Shultz, C. Linder, and C. ChoissyRossy for their critical comments. Dr. H.M. Dosch (Hospital for Sick Children, University of Toronto) is especially thanked for his wise counsel with regard to wording choices and the reminder that football in most of the world is played with the feet and not the hands. Drs. W. Frankel and C. Lutz of The Jackson Laboratory are thanked for providing pancreas samples and serum from their backcross mice, and Mr. W. Schott from the author's laboratory is thanked for his technical assistance. Dr. B. Richards-Smith and Ms. M. Berry are thanked for their assistance with nomenclature. The writing was supported by NIH grants DK36175, DK27722, DK56853, DK44240, and grants from The Juvenile Diabetes Research Foundation, International, and the American Diabetes Association. Institutional shared services at The Jackson Laboratory were supported by National Cancer Institute Cancer Center Support Grant CA-34 196. All studies reported herein that were carried out at The Jackson Laboratory followed the guidelines and regulations established by the institution's Animal Care and Use Committee.

\section{References}

1. Paigen K A miracle enough: the power of mice. Nat Med 1: 215-220

2. Sharp JJ, Linder CC, Mobraaten LE (2001) Genetically engineered mice. Husbandry and resources. Methods Mol Biol158: 381-396

3. Simpson EM, Linder CC, Sargent EE, Davisson MT, Mobraaten LE, Sharp JJ (1997) Genetic variation among 129 substrains and its importance for targeted mutagenesis in mice. Nat Genet 16: 19-27

4. Simpson E, Linder C, Sargent E, Davisson M, Mobraaten L, Sharp J (1997) Genetic variation among 129 substrains and its importance for targeted mutagenesis in mice. Nat Genet 16: 19-27

5. Threadgill DW, Yee D, Matin A, Nadeau JH, Magnuson T (1997) Genealogy of the 129 inbred strains: $129 / \mathrm{SvJ}$ is a contaminated inbred strain. Mamm Genome 8: 390-393

6. Mohrs M, Shinkai K, Mohrs K, Locksley RM (2001) Analysis of type 2 immunity in vivo with a bicistronic IL-4 reporter. Immunity 15: 303-311 
7. Ledermann B, Burki K (1991) Establishment of a germline competent C57BL/6 embryonic stem cell line. Exp Cell Res 197: 254-258

8. Yagi T, Tokunaga T, Furuta Y et al. (1993) A novel ES cell line, TT2, with high germline-differentiating potency. Anal Biochem 214: 70-76

9. Nagafuchi S, Katsuta H, Kogawa K et al. (1999) Establishment of an embryonic stem (ES) cell line derived from a non- obese diabetic (NOD) mouse: in vivo differentiation into lymphocytes and potential for germ line transmission. FEBS Lett 455: 101-104

10. Sima AAF, Shafrir E (2001) Animal Models of Diabetes: A Primer. Harwood Academic Publishers, Amsterdam

11. Coleman DL (1978) Obese and diabetes: two mutant genes causing diabetes-obesity syndromes in mice. Diabetologia 14: $141-148$

12. Beisel WR (1982) Single nutrients and immunity. Am J Clin Nutr 35 [Suppl]: 417-468

13. Leiter EH, Reifsnyder PC, Flurkey K, Partke H-J, Junger E, Herberg L (1998) Non-insulin dependent diabetes genes in mice: deleterious synergism by both parental genomes contributes to diabetogenic thresholds. Diabetes 47: 1287-1295

14. Stoehr JP, Nadler ST, Schueler KL et al. (2000) Genetic obesity unmasks nonlinear interactions between murine type 2 diabetes susceptibility loci. Diabetes 49: 1946-1954

15. McAleer M, Reifsnyder P, Palmer S et al. (1995) Crosses of NOD mice with the related NON strain: a polygenic model for type I diabetes. Diabetes 44: 1168-1195

16. Wicker LS, Todd JA, Peterson LB (1995) Genetic control of autoimmune diabetes in the NOD mouse. Ann Rev Immunol 13: 179-200

17. Hattori M, Yamato E, Matsumoto E et al. (1996) Occurrence of pretype I diabetes (pre-IDDM) and type II diabetes (NIDDM) in BC1 [(NOD x Mus spretus)F1 x NOD] mice. In: Shafrir E (ed) Lessons from Animal Diabetes Vol 6. Birkhaüser, Boston. pp 83-95

18. Leiter EH, Coleman DL, Eisenstein AB, Strack I (1980) $d b^{3 J}$, a new mutation at the diabetes locus in strain 129/J mice. I. Physiological and histological characterization. Diabetologia 19: 58-65

19. Kaku K, Province M, Permutt M (1989) Genetic analysis of obesity-induced diabetes associated with a limited capacity to synthesize insulin in C57BL/Ks mice: evidence for polygenic control. Diabetologia 32: 636-643

20. Accili D, Drago J, Lee EJ et al. (1996) Early neonatal death in mice homozygous for a null allele of the insulin receptor gene. Nat Genet 12: 106-109

21. Kido Y, Philippe N, Schaffer AA, Accili D (2000) Genetic modifiers of the insulin resistance phenotype in mice. Diabetes 49: 589-596

22. Leiter EH, Herberg L (1997) The polygenetics of diabesity in mice. Diabetes Rev5: 131-148

23. Bruning JC, Winnay J, Bonner-Weir S, Taylor SI, Accili D, Kahn CR (1997) Development of a novel polygenic model of NIDDM in mice heterozygous for IR and IRS-1 null alleles. Cell 88: 561-572

24. Kido Y, Burks DJ, Withers D et al. (2000) Tissue-specific insulin resistance in mice with mutations in the insulin receptor, IRS-1, and IRS-2. J Clin Invest 105: 199-205

25. Cahill G, Jones E, Lauris V, Steinke J, Soeldner J (1967) Studies on experimental diabetes in the Wellesley hybrid mouse. II. Serum insulin levels and response of peripheral tissues. Diabetologia 3: 171-174

26. Ranheim T, Dumke C, Schueler KL, Cartee GD, Attie AD (1997) Interaction between BTBR and C57BL/6 J genomes produces an insulin resistance syndrome in (BTBR $x$
C57BL/6 J) F1 mice. Arterioscler Thromb Vasc Biol 17: 3286-3293

27. Leiter EH (1988) Control of spontaneous glucose intolerance, hyperinsulinemia, and islet hyperplasia in nonobese C3H.SW male mice by Y-linked locus and adrenal gland. Metabolism 37: 689-696

28. Frankel WN, Johnson EW, Lutz CM (1995) Congenic strains reveal effects of the epilepsy quantitative trait locus, El2, separate from other El loci. Mamm Genome 6: 839-843

29. Faustman D, Li X, Lin HY et al. (1992) Linkage of faulty major histocompatibility complex class I to autoimmune diabetes. Science 254: 1756-1761

30. Serreze DV, Leiter EH, Christianson GJ, Greiner D, Roopenian DC (1994) MHC class I deficient NOD- $B 2 m^{\text {null }}$ mice are diabetes and insulitis resistant. Diabetes 43: 505-509

31. Wicker LS, Leiter EH, Todd JA et al. (1994) B2 microglobulin-deficient NOD mice do not develop insulitis or diabetes. Diabetes 43: 500-504

32. Katz J, Benoist C, Mathis D (1993) Major histocompatibility complex class I molecules are required for the development of insulitis in non-obese diabetic mice. Eur J Immunol 23: 3358-3360

33. Kim JK, Zisman A, Fillmore JJ et al. (2001) Glucose toxicity and the development of diabetes in mice with musclespecific inactivation of GLUT4. J Clin Invest 108: 153-160

34. Lund FE, Muller-Steffner HM, Yu N, Stout CD, Schuber F, Howard MC (1999) CD38 signaling in B lymphocytes is controlled by its ectodomain but occurs independently of enzymatically generated ADP-ribose or cyclic ADP-ribose. J Immunol 162: 2693-2702

35. Okamoto H (1999) The CD38-cyclic ADP-ribose signaling system in insulin secretion. Mol Cell Biochem 193: 115-118

36. Kato I, Yamamoto Y, Fujimura M, Noguchi N, Takasawa S, Okamoto H (1999) CD38 disruption impairs glucose-induced increases in cyclic ADP-ribose, $[\mathrm{Ca} 2+] \mathrm{i}$, and insulin secretion. J Biol Chem 274: 1869-1872

37. Ueda H, Ikegami H, Yamato E et al. (1995) The NSY mouse: A new animal model of spontaneous NIDDM with moderate obesity. Diabetologia 39: 503-508

38. Mathews CM, Bagley R, Caldwell J, Leiter EH (2001) NIDDM in a new mouse model elicited by low free radical scavenging potential combined with behaviorial and dietary stress. Diabetes 50 [Suppl 1]: A517 (Abstract)

39. Pelleymounter MA, Cullen MJ, Healy D, Hecht R, Winters D, McCaleb M (1998) Efficacy of exogenous recombinant murine leptin in lean and obese 10- to 12-mo-old female CD-1 mice. Am J Physiol 275: R950-R959

40. Hill NJ, Lyons PA, Armitage N, Todd JA, Wicker LS, Peterson LB (2000) The NOD Idd5 locus controls insulitis and diabetes and overlaps the orthologous CTLA4/IDDM 12 and NRAMP1 loci in humans. Diabetes 49: 1744-1747

41. Hultgren B, Huang XJ, Dybdal N, Stewart TA (1996) Genetic absence of gamma-interferon delays but does not prevent diabetes in NOD mice. Diabetes 45: 812-817

42. Serreze DV, Chapman HD, Post CM, Johnson EA, SuarezPinzon WL, Rabinovitch A (2001) Th1 to Th2 cytokine shifts in Nonobese Diabetic Mice: sometimes an outcome rather than the cause of diabetes resistance elicited by immunostimulation. J Immunol 166: 1352-1359

43. Wang B, Andre I, Gonzalez A et al. (1997) Interferon-gamma impacts at multiple points during the progression of autoimmune diabetes. Proc Natl Acad Sci $U S A$ 94: 13844-13849

44. Kanagawa O, Xu G, Tevaarwerk A, Vaupel BA (2000) Protection of nonobese diabetic mice from diabetes by gene(s) closely linked to IFN-gamma receptor loci. J Immunol 164: 3919-3923 
45. Serreze DV, Post CM, Chapman HD, Johnson EA, Lu B, Rothman PB (2000) Interferon-gamma receptor signaling is dispensable in the development of autoimmune type $1 \mathrm{di}-$ abetes in NOD mice. Diabetes 49: 2007-2011

46. Bristol IJ, Cong Y, Farmer MA et al. (2000) Heritable susceptibility to colitis in mice induced by interleukin-10 deficiency. Inflamm Bowel Dis 6: 290-302

47. Bruning JC, Michael MD, Winnay JN et al. (1998) A muscle-specific insulin receptor knockout exhibits features of the metabolic syndrome of NIDDM without altering glucose tolerance. Mol Cell 2: 559-569

48. Nakae J, Barr V, Accili D (2000) Differential regulation of gene expression by insulin and IGF-1 receptors correlates with phosphorylation of a single amino acid residue in the forkhead transcription factor FKHR. EMBO J 19: 989-996

49. Shall S, de Murcia G (2000) Poly(ADP-ribose) polymerase-1: what have we learned from the deficient mouse model? Mutat Res 460: 1-15

50. Burnett AL, Nelson RJ, Calvin DC et al. (1996) Nitric oxide-dependent penile erection in mice lacking neuronal nitric oxide synthase. Mol Med 2: 288-296

51. Liu SC, Wang Q, Lienhard GE, Keller SR (1999) Insulin receptor substrate 3 is not essential for growth or glucose homeostasis. J Biol Chem 274: 18093-18099

52. Hamann A, Flier JS, Lowell BB (1996) Decreased brown fat markedly enhances susceptibility to diet-induced obesity, diabetes, and hyperlipidemia. Endocrinology 137: 21

53. Leiter EH (1990) The role of environmental factors in modulating insulin dependent diabetes. In: de Vries R, Cohen I, van Rood J, (eds) Current Topics in Immunology and Microbiology. The Role of Microorganisms in Non-infectious Disease. Springer Verlag, Berlin. pp 39-55

54. Leiter EH (1998) NOD mice and related strains: origins, husbandry, and biology. In: Leiter EH, Atkinson MA, (eds) NOD Mice and Related Strains: Research Applications in Diabetes, AIDS, Cancer, and Other Diseases. R. G. Landes, Austin. pp 1-35

55. Atkinson MA, Leiter EH (1999) The NOD mouse model of type 1 diabetes: as good as it gets? Nat Med 5: 601-604

56. Olson EN, Arnold HH, Rigby PW, Wold BJ (1996) Know your neighbors: three phenotypes in null mutants of the myogenic bHLH gene MRF4. Cell 85: 1-4

57. Fiering S, Epner E, Robinson K (1995) et al. Targeted deletion of 5'HS2 of the murine beta-globin LCR reveals that it is not essential for proper regulation of the beta-globin locus. Genes Dev 9: 2203-2213

58. Xu H, Shields D (1994) Prosomatostatin processing in permeabilized cells. Endoproteolytic cleavage is mediated by a vacuolar ATPase that generates an acidic $\mathrm{pH}$ in the trans-Golgi network. J Biol Chem 269: 22875-22881

59. Hormuzdi SG, Penttinen R, Jaenisch R, Bornstein P (1988) A gene-targeting approach identifies a function for the first intron in expression of the alpha1(I) collagen gene. Mol Cell Biol 18: 3368-3375

60. Miles PDG, Barak Y, He WM, Evans RM, Olefsky JM (2000) Improved insulin-sensitivity in mice heterozygous for PPAR-gamma deficiency. J Clin Invest 105: 287-292

61. Kundig TM, Schorle H, Bachmann MF, Hengartner H, Zinkernagel RM, Horak I (1993) Immune responses in interleukin-2-deficient mice. Science 262: 1059-1061

62. Sadlack B, Merz H, Schorle H, Schimpl A, Feller AC, Horak I (1993) Ulcerative colitis-like disease in mice with a disrupted interleukin-2 gene. Cell 75: 253-261

63. Chattopadhyay SK, Morse HCD, Makino M, Ruscetti SK, Hartley JW (1989) Defective virus is associated with induc- tion of murine retrovirus-induced immunodeficiency syndrome. Proc Natl Acad Sci U S A 86: 3862-3866

64. Allison J, Harrison LC, Campbell IL, Miller JFAP (1990) Major histocompatibility complex molecules and the betacell: inferences from transgenic mice. Curr Topics Microbiol Immunol 156: 121-135

65. Hanson MS, Cetkovic-Cvrlje M, Ramiya V et al. (1996) Quantitative thresholds of MHC Class II I-E expression on hematopoietically derived APC in transgenic NOD/Lt Mice determine level of diabetes resistance and indicate mechanism of protection. J Immunol 157: 1279-1287

66. Bridgett MM, Cetkovic-Cvrlje M, Narayanswami S (1998) et al. Differential protection in two transgenic lines of NOD/Lt mice hyperexpressing the autoantigen GAD65 in pancreatic beta cells. Diabetes 47: 1848-1856

67. Leiter E (1997) The NOD mouse: a model for insulin dependent diabetes mellitus. In: Coligan JE, Kruisbeek AM, Margulies DM, Shevach EM, Strober W (eds) Current Protocols in Immunology. John Wiley \& Sons, New York. pp 15.9.1-15.9.23

68. Yoon JW, Yoon CS, Lim Hw et al. (1999) Control of autoimmune diabetes in NOD mice by GAD expression or suppression in B cells. Science 284: 1183-1186

69. Tian J, Chau C, Hales TG, Kaufman DL (1999) GABA(A) receptors mediate inhibition of $\mathrm{T}$ cell responses. J Neuroimmunol 96: 21-28

70. Winer S, Astsaturov I, Cheung R et al. (2001) Type I diabetes and multiple sclerosis patients target islet plus central nervous system autoantigens; nonimmunized nonobese diabetic mice can develop autoimmune encephalitis. J Immunol 166: 2831-2841

71. Salomon B, Rhee L, Bour-Jordan H et al. (2001) Development of spontaneous autoimmune peripheral polyneuropathy in B7-2- deficient NOD mice. J Exp Med 194: 677-684

72. Serreze DV, Chapman HD, Varnum DS, Hanson MS, Reifsnyder PC, Richard SD et al. (1996) B lymphocytes are essential for the initiation of T cell-mediated autoimmune diabetes: analysis of a new "speed congenic" stock of NOD.Ig $\mu^{\text {null }}$ mice. J Exp Med 184: 2049-2053

73. Postic C, Shiota M, Niswender KD et al. (1999) Dual roles for glucokinase in glucose homeostasis as determined by liver and pancreatic beta cell-specific gene knock-outs using Cre recombinase. J Biol Chem 274: 305-315

74. Mauvais-Jarvis F, Virkamaki A, Michael MD et al. (2000) A model to explore the interaction between muscle insulin resistance and beta-cell dysfunction in the development of type 2 diabetes. Diabetes 49: 2126-2134

75. Niwa-Kawakita M, Abramowski V, Kalamarides M, Thomas G, Giovannini M (2000) Targeted expression of Cre recombinase to myelinating cells of the central nervous system in transgenic mice. Genesis 26: 127-129

76. Schmidt EE, Taylor DS, Prigge JR, Barnett S, Capecchi MR (2000) Illegitimate Cre-dependent chromosome rearrangements in transgenic mouse spermatids. Proc Natl Acad Sci U S A 97: 13702-13707

77. Loonstra A, Vooijs M, Beverloo HB et al. (2001) Growth inhibition and DNA damage induced by Cre recombinase in mammalian cells. Proc Natl Acad Sci U S A 98: 9209-9214

78. Thomas MK, Devon ON, Lee JH et al. (2001) Development of diabetes mellitus in aging transgenic mice following suppression of pancreatic homeoprotein IDX-1. J Clin Invest 108: 319-329 